\title{
Multiquantum Chemical Exchange Saturation Transfer NMR to Quantify Symmetrical Exchange: Application to Rotational Dynamics of the Guanidinium Group in Arginine Side Chains
}

\author{
Gogulan Karunanithy, Jochen Reinstein, and D. Flemming Hansen* \\ Cite This: J. Phys. Chem. Lett. 2020, 11, 5649-5654 \\ Read Online
}

ACCESS | W Metrics \& More 回 Article Recommendations

ABSTRACT: Chemical exchange saturation transfer (CEST) NMR experiments have emerged as a powerful tool for characterizing dynamics in proteins. We show here that the CEST approach can be extended to systems with symmetrical exchange, where the NMR signals of all exchanging species are severely broadened. To achieve this, multiquantum CEST (MQ-CEST) is introduced, where the CEST pulse is applied to a longitudinal multispin order density element and the CEST profiles are encoded onto nonbroadened nuclei. The MQCEST approach is demonstrated on the restricted rotation of guanidinium groups in arginine residues within proteins. These groups and their dynamics are essential for many enzymes and for noncovalent interactions through the formation of hydrogen bonds, salt-bridges, and $\pi$ stacking interactions, and their rate of rotation is highly indicative of the extent of interactions formed. The MQ-CEST method is successfully applied to guanidinium groups in the $19 \mathrm{kDa}$ L99A mutant of T4 lysozyme.

\begin{abstract}
A key strength of NMR spectroscopy is its ability to $\mathcal{1}_{\text {quantify the dynamics of molecules with atomic level }}$ resolution. Within biomolecules, conformational exchanges often occur on milli- to microsecond time scales, and these exchanges can be critical for function. ${ }^{1,2}$ A number of NMRbased approaches for characterizing exchange on these time scales now exist and provide important insights into conformations that are transiently populated, invisible to other high-resolution methods, and also broadened beyond detection in traditional NMR experiments. ${ }^{3-6}$ Although chemical exchange saturation transfer (CEST) methods have traditionally been used within the MRI field, ${ }^{7-9}$ CEST approaches have recently emerged as powerful tools also for studying biomolecular dynamics on a time scale from 20 to $200 \mathrm{~s}^{-1} \cdot{ }^{10,11}$ In these experiments, first developed in the $1960 \mathrm{~s}^{12}$ saturation of magnetization by a weak pulse is transferred by exchange events within a network of exchanging conformers, and in particular, magnetization is transferred from invisible species to visible species in order to report on chemical shifts and rates of exchange.

However, for symmetrical exchange, where all the exchanging species are broadened in NMR spectra, a quantification of the exchanging system becomes challenging. This scenario is, for example, encountered for the rotational exchange about the $\mathrm{C}^{\zeta}-\mathrm{N}^{\varepsilon}$ bond in arginine side chains in proteins, Figure 1A. The importance of the arginine side chain for a range of protein functions, such as protein folding, catalysis, and noncovalent interactions, is well-established. ${ }^{13-15}$ It is the arginine guanidinium group, with its delocalized $\pi$ system, that confers the functionality by allowing for a large
\end{abstract}

range of interactions. ${ }^{16-18}$ Recently, it was shown that ${ }^{13} \mathrm{C}$ detection NMR provides an excellent tool to probe arginine side chains in proteins, ${ }^{19-22}$ and it was also shown that in favorable cases the rate of rotational exchange can be determined to provide a measure for the interactions formed by arginine guanidinium groups within proteins. ${ }^{23}$ Below, we present a multiquantum CEST (MQ-CEST) NMR experiment that is ideally suited to characterizing dynamics in symmetrically exchanging groups, and we apply this methodology to quantify the rotational dynamics of guanidinium groups in the side chains of arginine residues within proteins.

In the multiquantum CEST (MQ-CEST) experiment, the CEST pulse is applied to a longitudinal multispin order spindensity element in order to quantify symmetrical exchange. The restricted rotation about the $\mathrm{C}^{\zeta}-\mathrm{N}^{\varepsilon}$ bond in arginine side chains, Figure 1A, corresponds to a symmetrical exchange of the two ${ }^{15} \mathrm{~N}^{\eta}$ nuclei. The MQ-CEST approach applied to a density operator that spans both of the ${ }^{15} \mathrm{~N}^{\eta}$ species is able to capture the rate of restricted rotation as well as the chemical shifts of the sometimes severely broadened ${ }^{15} \mathrm{~N}^{\eta}$ signals.

For the MQ-CEST approach applied to arginine guanidinium side chains, two equally sized dips are typically observed corresponding to the CEST pulse being resonant with one of

Received: April 30, 2020

Accepted: June 16, 2020

Published: June 16, 2020 
A

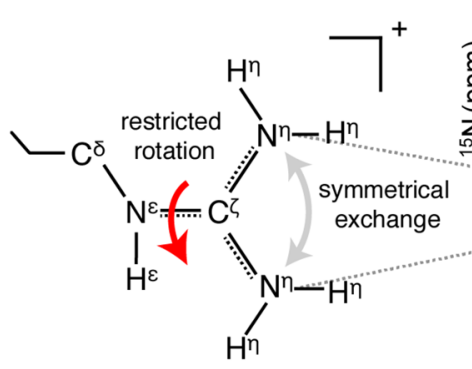

B

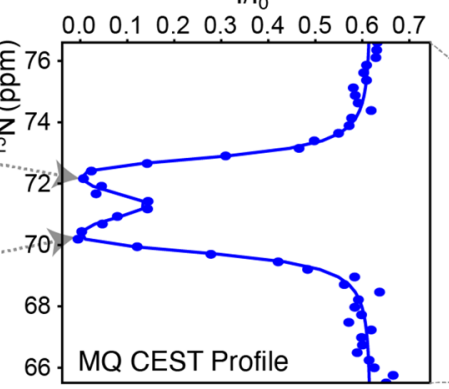

C

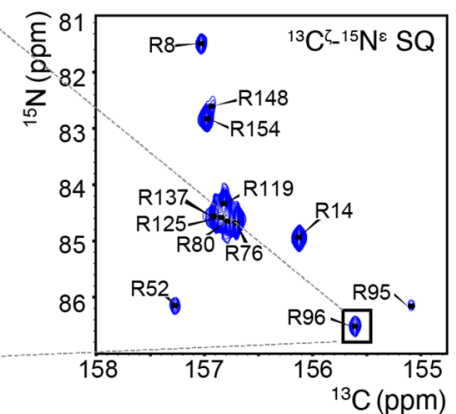

D

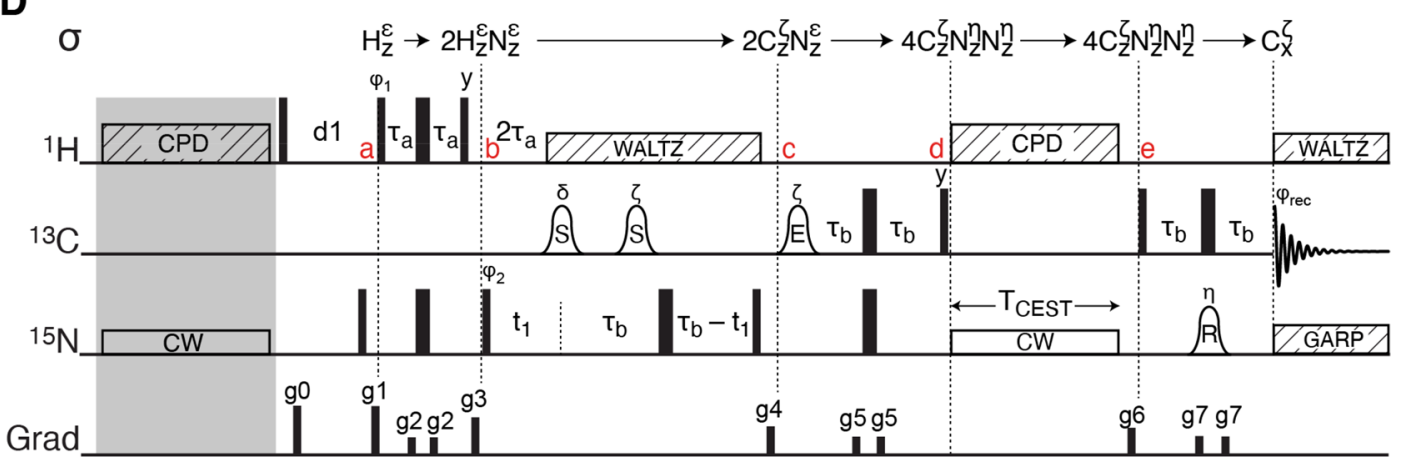

Figure 1. (A) Illustration of an arginine side chain and the restricted rotation about the $\mathrm{C}^{\zeta}-\mathrm{N}^{\varepsilon}$ bond that causes the symmetrical exchange of the two ${ }^{15} \mathrm{~N}^{\eta}$ nuclei. (B) Example of a ${ }^{15} \mathrm{~N}^{\eta}-{ }^{15} \mathrm{~N}^{\eta}$ MQ-CEST profile, recorded for the R96 arginine in the L99A mutant of T4 lysozyme (T4L99A). The position of the two dips shows the ${ }^{15} \mathrm{~N}^{\eta}$ chemical shifts, and a least-squares fit to the profile provides the rate of exchange. (C) Example of a ${ }^{13} \mathrm{C}^{\zeta}-{ }^{15} \mathrm{~N}$ correlation map for T4L99A that encodes the CEST profiles. (D) Pulse sequence derived for the MQ-CEST experiment applied to arginine side chains. The carrier frequencies are set to $7.15 \mathrm{ppm}\left({ }^{1} \mathrm{H}\right), 157 \mathrm{ppm}\left({ }^{13} \mathrm{C}\right)$, and $84 \mathrm{ppm}\left({ }^{15} \mathrm{~N}\right)$. Narrow and wide bars denote $90^{\circ}$ and $180^{\circ}$ pulses, respectively, with all pulses applied along $x$ unless otherwise indicated. The bell shapes denote frequency-selective pulses, with the letter above showing selectivity and letter inside indicating the type: $S$ refers to a Seduce shape, ${ }^{24} \mathrm{E}$ to an EBURP shape, and R to a REBURP shape. ${ }^{25}$ Striped boxes indicate decoupling with the text indicating the scheme used. CW: continuous wave applied at CEST offset frequency. The delays are $\tau_{\mathrm{a}}={ }^{1} /\left(4 \mathrm{~J}_{\mathrm{HN}}\right)=2.7 \mathrm{~ms}$ and $\tau_{\mathrm{b}}=1 /\left(4 J_{\mathrm{NC}}\right)=12.5 \mathrm{~ms}$. The phase cycle used is $\phi_{1}=x,-x ; \phi_{2}=2(x), 2(-x) ;$ and $\phi_{\mathrm{rec}}=x,-x,-x, x$. Gradients are used to remove artifacts (full details in the caption to Figure S4).

the two ${ }^{15} \mathrm{~N}^{\eta}$ chemical shifts, Figure $1 \mathrm{~B}$ and Figure S1. When the exchanging nuclei are in the so-called slow-exchange regime $\left(k_{\text {ex }} \ll\left|\omega\left({ }^{15} \mathrm{~N}^{\eta 1}\right)-\omega\left({ }^{15} \mathrm{~N}^{\eta 2}\right)\right|=|\Delta \omega|\right),{ }^{26,27}$ two wellseparated dips are visible in the MQ-CEST profile and the individual chemical shift of the two ${ }^{15} \mathrm{~N}^{\eta}$ nuclei can be directly identified from the center of the dips, Figure 1B and Figure S1. When the nuclei are in the intermediate chemical exchange regime $\left(k_{\mathrm{ex}} \approx|\Delta \omega|\right)$, either because of fast rotation or because of a smaller chemical shift difference, the two dips coalesce into a single dip centered at the average of the two ${ }^{15} \mathrm{~N}^{\eta}$ chemical shifts. It is particularly near the intermediate chemical exchange regime that single-quantum ${ }^{15} \mathrm{~N}^{\eta}$ signals become severely broadened in NMR spectra. ${ }^{22}$ For the MQ-CEST approach, the CEST intensities are encoded onto ${ }^{13} \mathrm{C}^{\zeta}-{ }^{15} \mathrm{~N}^{\varepsilon}$ correlation maps that are unaffected by the rotational exchange. Therefore, as long as CEST offset frequencies are chosen to fully cover the CEST $\operatorname{dip}(\mathrm{s})$, the exchanging system can be characterized using the MQ-CEST approach, even for scenarios of intermediate exchange. When the rotation is in the fast-exchange regime $\left(k_{\mathrm{ex}} \gg|\Delta \omega|\right)$, the width of the single CEST dip narrows.

Simulations were used to establish the range of parameters, where accurate exchange parameters, $k_{\mathrm{ex}}$ and $\Delta \omega$, can be derived using the MQ-CEST approach. In common with other CEST approaches, the MQ-CEST approach provides the most accurate parameters, when there is a substantial chemical shift difference between the two exchanging ${ }^{15} \mathrm{~N}^{\eta}$ species, which brings the system toward the slow-exchange regime. The simulations show that, for $\Delta \omega \geq 1 \mathrm{ppm}$, the MQ-CEST approach provides accurate exchange rates over the large range of $k_{\text {ex }}$ from 5 to $1500 \mathrm{~s}^{-1}$, which covers almost the full range of possible arginine guanidinium rotation rates at $293 \mathrm{~K}$. Generally, the larger the chemical shift differences are, $\Delta \omega$, the larger exchange rates $k_{\text {ex }}$ are accessible, Figure S2, whereas accurate $\Delta \omega$ values can be obtained for the full range of possible $k_{\text {ex }}$ values from 5 to $1500 \mathrm{~s}^{-1}$, Figure S3, as long as $\Delta \omega$ $\gtrsim 0.5 \mathrm{ppm}$. For arginine side chains involved in very strong interactions and thus experiencing very slow rotational rates $\left(<5 \mathrm{~s}^{-1}\right)$, it is possible to set an upper bound for $k_{\mathrm{ex}}$ and accurately determine the two ${ }^{15} \mathrm{~N}^{\eta}$ chemical shifts from MQCEST experiments. In such cases of very slow exchange, the $k_{\mathrm{ex}}$ can be determined using the previous longitudinal exchange method, ${ }^{23}$ provided that the ${ }^{13} \mathrm{C}^{\zeta}$ resonance is isolated. It is interesting to note that the range of chemical exchange rates, $k_{\mathrm{ex}}$, accessible with the MQ-CEST approach is substantially larger than what is accessible from typical CEST experiments ${ }^{7,8}$ $\left(20-200 \mathrm{~s}^{-1}\right)$. This larger range of $k_{\mathrm{ex}}$ accessible with the MQCEST approach is mainly due to the symmetrical exchange with equal populations of the two exchanging sites. It is also important to note that with least-squares fitting (see Supporting Information) and detailed analysis of the MQCEST profiles it is possible to accurately extract the chemical shifts of the two ${ }^{15} \mathrm{~N}^{\eta}$ nuclei well beyond the range where these 
can be simply obtained from inspection of the MQ-CEST profiles.

Finally, the simulations show that it is highly desirable to collect data with different $B_{1}$ CEST saturation field strengths and/or at different static magnetic field strengths, $B_{0}$, Figures S2 and S3. It is interesting to note that MQ-CEST profiles with multiple $B_{1}$ field strengths provide essentially as accurate $k_{\text {ex }}$ rates and $\Delta \omega$ as MQ-CEST profiles at multiple $B_{0}$ fields. Recording MQ-CEST profiles at multiple static $B_{0}$ fields, particularly at higher field strengths, gives access to higher rotational rates and smaller chemical shift differences, since the exchange is moved toward the slow-chemical exchange regime. A disadvantage of ultrahigh magnetic fields $\left(B_{0} \gtrsim 19 \mathrm{~T}\right)$, however, is an increased ${ }^{13} \mathrm{C}^{\zeta}$ transverse relaxation due to chemical shift anisotropy (CSA), which can reduce signal-tonoise in the resulting spectra.

The pulse sequence derived for obtaining MQ-CEST profiles to characterize the symmetric exchange of arginine guanidinium groups in proteins is shown in Figure 1D. Briefly, equilibrium longitudinal magnetization residing on ${ }^{1} \mathrm{H}^{\varepsilon}, H_{z}^{\varepsilon}$, is initially excited and transferred via an INEPT step between $a$ and $b$ to a ${ }^{1} \mathrm{H}^{\varepsilon}-{ }^{15} \mathrm{~N}^{\varepsilon}$ longitudinal two-spin order density element, $2 H_{z}^{\varepsilon} N_{z}^{\varepsilon}$. Using the magnetization on the ${ }^{1} \mathrm{H}^{\varepsilon}$ proton as the source brings two main advantages compared to methods where ${ }^{13} \mathrm{C}$ magnetization is used as the source. First, the higher gyromagnetic ratio of ${ }^{1} \mathrm{H}$ provides additional signal-to-noise, even though this is partly mitigated by the longer sequence. Second, ${ }^{1} \mathrm{H}$ nuclei have substantially faster longitudinal $R_{1}$ relaxation rates compared to ${ }^{13} \mathrm{C}$, which means that more scans can be acquired within a given time unit. Between $b$ and $c$, the one-bond scalar coupling between ${ }^{1} \mathrm{H}^{\varepsilon}$ and ${ }^{15} \mathrm{~N}^{\varepsilon}$ and between ${ }^{15} \mathrm{~N}^{\varepsilon}$ and ${ }^{13} \mathrm{C}^{\zeta}$ is evolved to generate the ${ }^{13} \mathrm{C}^{\zeta}-{ }^{15} \mathrm{~N}^{\varepsilon}$ longitudinal two-spin order element $2 C_{z}^{\zeta} N_{z}^{\varepsilon}$, while concomitantly encoding the ${ }^{15} \mathrm{~N}^{\varepsilon}$ chemical shift. The two selective ${ }^{13} \mathrm{C}$ pulses between $b$ and $c$ ensure that ${ }^{13} \mathrm{C}^{\delta}-{ }^{15} \mathrm{~N}^{\varepsilon}$ and ${ }^{13} \mathrm{C}^{\zeta}-{ }^{15} \mathrm{~N}^{\varepsilon}$ scalar couplings are refocused and evolved, respectively. Between $c$ and $d$, a further INEPT is used to evolve ${ }^{13} \mathrm{C}^{\zeta}-{ }^{15} \mathrm{~N}^{\varepsilon}$ and ${ }^{13} \mathrm{C}^{\zeta}-{ }^{15} \mathrm{~N}^{\eta}$ scalar couplings, yielding a density element proportional to $4 C_{z}^{\zeta} N_{z}^{\eta} N_{z}^{\eta}$ at point $d$. The MQ-CEST period between points $d$ and $e$ is carried out with the ${ }^{15} \mathrm{~N}$ carrier frequency being varied, providing the CEST intensities, I. A reference spectrum is also recorded without the CEST element $\left(T_{\text {CEST }}=0 \mathrm{~s}\right)$, but including the gray block in Figure 1D. The reference spectrum provides $I_{0}$ and the final MQCEST profiles are calculated as $I / I_{0}$. The effects of scalar couplings between ${ }^{15} \mathrm{~N}^{\eta}$ and ${ }^{1} \mathrm{H}$ are minimized through the application of high-power composite decoupling during the CEST period as described previously. ${ }^{28}$ As the ${ }^{15} \mathrm{~N}$ CEST pulse is applied to a $4 C_{z}^{\zeta} N_{z}^{\eta} N_{z}^{\eta}$ density element, no decoupling is applied to ${ }^{13} \mathrm{C}$ as this would deteriorate the signal. Instead, the effects of the ${ }^{13} \mathrm{C}^{\zeta}-{ }^{15} \mathrm{~N}^{\eta}$ scalar couplings $(\sim 20 \mathrm{~Hz})$ are explicitly included in the analysis of the CEST profiles as described previously. ${ }^{21,29}$ It should be noted that several density elements are present during the ${ }^{15} \mathrm{~N}$ CEST pulse, including Zeeman order $\left(4 C_{z}^{\zeta} N_{z}^{\eta} N_{z}^{\eta}\right)$, zero-quantum (e.g., $4 C_{z}^{\zeta} N_{+}^{\eta} N_{-}^{\eta}$ ), single-quantum (e.g., $4 C_{z}^{\zeta} N_{z}^{\eta} N_{-}^{\eta}$ ), and doublequantum (e.g., $4 C_{z}^{\zeta} N_{+}^{\eta} N_{+}^{\eta}$ ) coherences. Whereas the doublequantum coherences are insensitive to the exchange, ${ }^{22}$ the zero-quantum coherence will report on the exchange process. Finally, after point $e$, the density element of interest is transferred to transverse in-phase ${ }^{13} \mathrm{C}^{\zeta}$ magnetization for detection.
Several variations to the pulse sequence in Figure 1D have been developed (Figure S4). Of particular note is the ${ }^{1} \mathrm{H}$ detected version (Figure S4B) in which magnetization, via additional INEPT steps, is transferred back to the ${ }^{1} \mathrm{H}^{\varepsilon}$ proton for detection. If relaxation is ignored, detecting ${ }^{1} \mathrm{H}^{\varepsilon}$ gives an 8fold increase in signal-to-noise compared to ${ }^{13} \mathrm{C}$ detection; however, in practice the additional delays required as well as exchange of ${ }^{1} \mathrm{H}^{\varepsilon}$ with the bulk solvent mean that this approach is only advantageous when the site in question has a local correlation time of less than approximately 10 ns (Figure S5). The guanidinium groups of greatest interest are often those that form interactions, making them more rigid, and so for the applications shown below on the $19 \mathrm{kDa}$ T4L99A, the ${ }^{13} \mathrm{C}$ detect sequence, Figure 1D, provides the best signal-to-noise.

In order to experimentally validate the MQ-CEST approach, experiments were carried out on a sample of free $\left[{ }^{13} \mathrm{C}_{6},{ }^{15} \mathrm{~N}_{4}\right]-\mathrm{L}-$ arginine at a high concentration $(50 \mathrm{mM})$ and dissolved in a $50 / 50 \%$ vol mixture of $\mathrm{H}_{2} \mathrm{O}$ and $\mathrm{MeOH}$ under acidic conditions. With this sample, experiments can be carried out at temperatures below $0{ }^{\circ} \mathrm{C}$, where the symmetrical exchange rate, $k_{\text {ex }}$, for free arginine is slow enough to be quantified by classical longitudinal exchange methods, such as zz-EXSY. ${ }^{30}$ This system therefore forms an ideal basis for validating and benchmarking the performance of the MQ-CEST approach.

The MQ-CEST profiles for free arginine measured at four temperatures between -15 and $2.4{ }^{\circ} \mathrm{C}$ are shown in Figure 2A. The chemical shifts of the ${ }^{15} \mathrm{~N}^{\eta}$ nuclei can be easily identified from the position of the dips at low temperatures, and the exchange rate can be obtained from a least-squares analysis at each temperature (see Supporting Information). The correlation between the obtained exchange rates, $k_{\mathrm{ex}}$, from MQ-CEST and from longitudinal exchange is excellent, Figure 2C, validating the MQ-CEST approach. It is important to note that the longitudinal exchange approach is only applicable when the ${ }^{15} \mathrm{~N}^{\eta}$ nuclei give rise to diagonal and cross-peaks in single-quantum NMR spectra and these peaks can be accurately quantified. This is not the requirement for the MQ-CEST approach, since the CEST intensities are encoded onto the ${ }^{13} \mathrm{C}^{\zeta}-{ }^{15} \mathrm{~N}^{\varepsilon}$ cross-peaks.

Having demonstrated the validity of the MQ-CEST approach, both theoretically and experimentally, for extracting the rate for symmetrical exchange of the arginine guanidinium group, we turned our attention to arginine side chains within the $19 \mathrm{kDa}$ L99A mutant of T4 lysozyme (T4L99A). T4 lysozyme is a challenging test case since a large range of exchange rates spanning more than 3 orders of magnitude have been observed. ${ }^{23}$ Previously, D-evolution and longitudinal exchange were used to characterize the rotational dynamics of arginine guanidinium groups in T4L99A; however, these measurements rely on single-quantum ${ }^{13} \mathrm{C}^{\zeta}-{ }^{15} \mathrm{~N}^{\eta}$ spectra, and only five of the 13 arginine residues in T4L99A could previously be characterized. On the contrary, the MQ-CEST approach relies on ${ }^{13} \mathrm{C}^{\zeta}-{ }^{15} \mathrm{~N}^{\varepsilon}$ (or ${ }^{1} \mathrm{H}^{\varepsilon}-{ }^{15} \mathrm{~N}^{\varepsilon}$ ) spectra, where well-separated cross-peaks are observed, e.g., Figure 1C. Thus, the MQ-CEST approach shows substantial improvements over the existing D-evolution method, since nearly every arginine residue can be resolved, resulting in an exchange rate for 11 out of the 13 arginine residues in T4L99A at $293 \mathrm{~K}$. Four of these MQ-CEST profiles are shown in Figure 3, while all data is provided in Figure S6 and Table S2. For the arginine residues, where $k_{\text {ex }}$ could previously be obtained from the Devolution approach, there is an excellent agreement with the rates derived from the MQ-CEST profiles. ${ }^{23}$ 

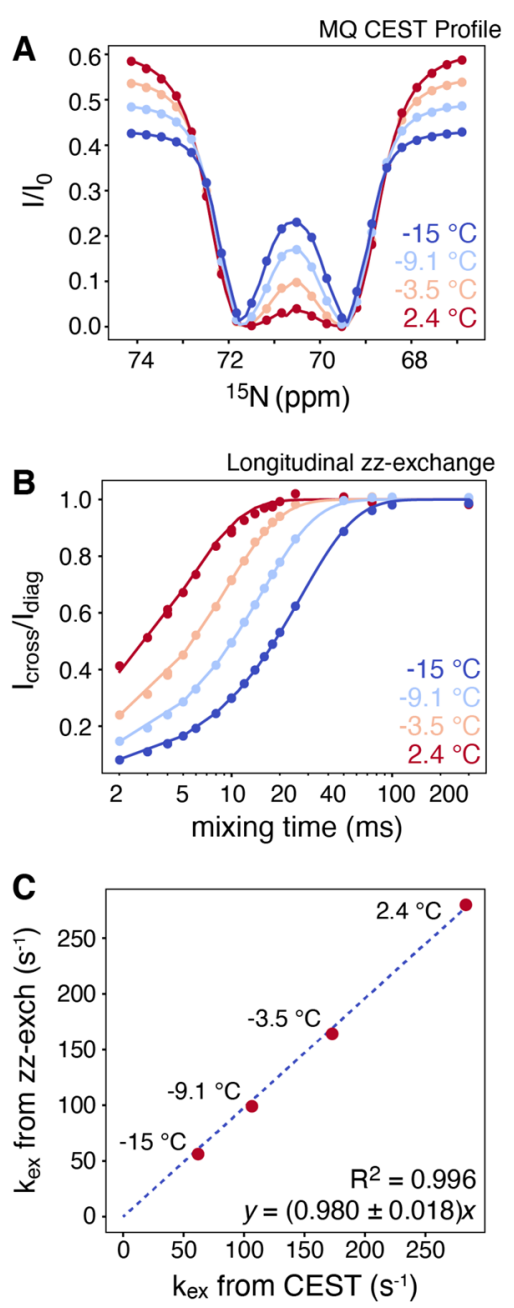

Figure 2. (A) MQ-CEST profiles recorded at four temperatures $(-15$ to $2.4{ }^{\circ} \mathrm{C}$ ) on a $50 \mathrm{mM}$ sample of $\left[{ }^{13} \mathrm{C}_{6},{ }^{15} \mathrm{~N}_{4}\right]$-L-arginine dissolved in $50 / 50 \% \mathrm{vol} \mathrm{H}_{2} \mathrm{O} / \mathrm{MeOH}$ at a static magnetic field of $14.1 \mathrm{~T}$ and using a $10 \mathrm{~Hz} B_{1}$ CEST saturation pulse for $T_{\mathrm{CEST}}=250 \mathrm{~ms}$. (B) Corresponding longitudinal exchange, zz-EXSY, data recorded on the same sample. (C) Correlation plot of symmetrical exchange rates obtained from MQ-CEST (abscissa) and from longitudinal exchange (ordinate). The excellent linear correlation between the rates shows that accurate rotational exchange rates, $k_{\mathrm{ex}}$ can be derived from the MQ-CEST experiment.

The obtained $k_{\mathrm{ex}}$ rates confirm that the rate of rotation is a very good indicator of interactions formed by a particular arginine side chain within the protein environment. For example, from the crystal structure of T4L99A, ${ }^{31}$ various interactions are observed for R52, R95, and R96, Figure 3, and these residues show a large range of rates, albeit all substantially slower than free arginine. The guanidinium group that shows the slowest rotational rate is in R95 that forms cation $-\pi$ and $\pi-\pi$ interactions with the large aromatic system of tryptophan W126.

In order to further assess the robustness of the MQ-CEST analysis for the extraction of exchange rates, the relaxation rates, $R\left(4 C_{z}^{\zeta} N_{y}^{\eta} N_{y}^{\eta}\right)$ and $R_{1}\left(C_{z}^{\zeta}\right)$, were measured experimentally for T4L99A. First, the experimentally measured relaxation rates were compared to the corresponding rates obtained from an analysis of the MQ-CEST profiles, where those rates were allowed to vary, however, independent of the static magnetic field (Figure S7). Second, two-dimensional $\chi^{2}\left(k_{\mathrm{ex}}, R_{2}\right)$ grid
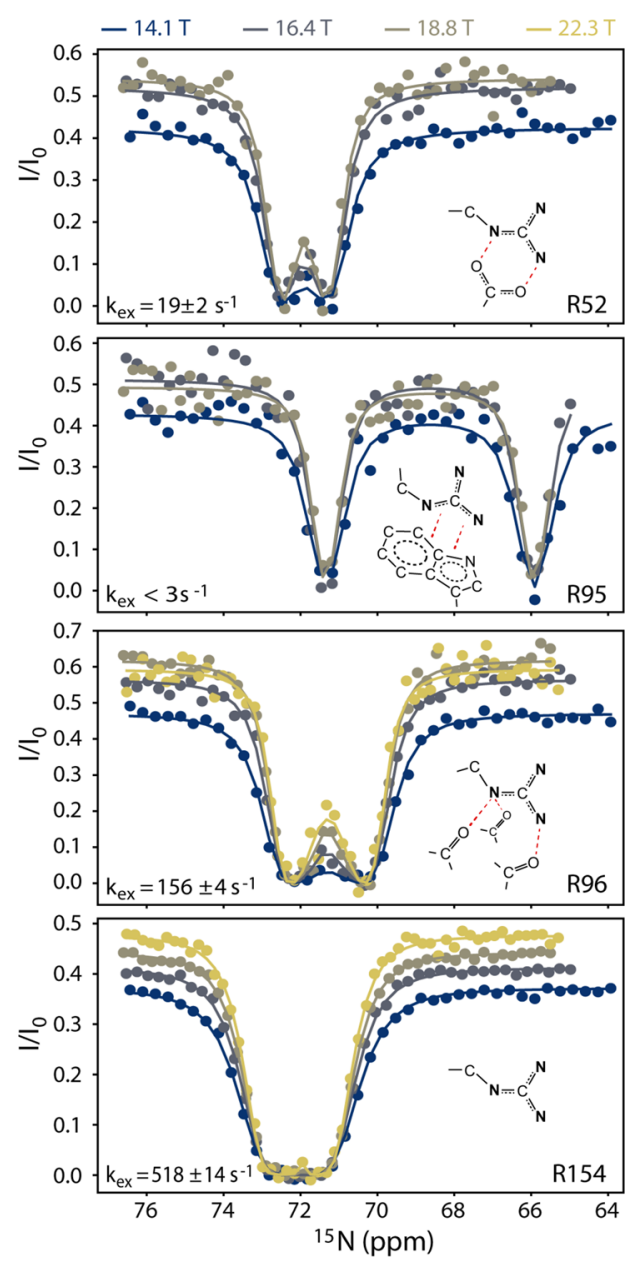

Figure 3. MQ-CEST profiles for arginine residues R52, R95, R96, and R154 of T4L99A. All data shown is collected at $293 \mathrm{~K}$ at static magnetic fields, $B_{0}$, between 14.1 and $22.3 \mathrm{~T}$. For a given arginine guanidinium group, all data is analyzed simultaneously to give the rate of symmetrical exchange, $k_{\mathrm{ex}}$ and the chemical shift of the two ${ }^{15} \mathrm{~N}^{\eta}$ nuclei.

plots were generated, Figure S8, to quantify the influence of the relaxation rates on the derived $k_{\mathrm{ex}}$. For nuclei with slower exchange rates, accurate transverse ${ }^{15} \mathrm{~N}^{\eta}$ relaxation rates can be obtained. In cases of faster rotational exchange rates, the exchange rate is uncorrelated with the transverse relaxation rate, Figure S8, meaning that the transverse relaxation rate can be safely fixed to a sensible value in the fitting process. In all cases, accurate rotational exchange rates, $k_{\mathrm{ex}}$ can be obtained (Figure S9).

In summary, we have described a multiquantum CEST NMR experiment, which is ideally suited for characterizing the rate of symmetrical exchange and the chemical shifts of the involved nuclei. The MQ-CEST approach was applied to quantify the rotation of guanidinium groups in arginine side chains in proteins, and it is shown that the MQ-CEST approach can accurately provide the rate of exchange over a very large range of time scales and also report on sites that previously remained undetected. The results confirm that the symmetrical rotational rate of arginine guanidinium groups within a protein environment is an insightful parameter reporting on the strength of interactions formed by the group. It is anticipated that the MQ-CEST methodology presented here will be generally applicable to quantify 
symmetric exchange over a large range of time scales and in many sites.

\section{EXPERIMENTAL METHODS}

Sample preparations are described in the Supporting Information. All spectra were processed using NMRPipe ${ }^{32}$ and visualized with NMRFAM-Sparky. ${ }^{33}$ Peak intensities were quantified using FuDa. ${ }^{34}$ MQ-CEST profiles were simulated and analyzed using an in-house program that numerically propagates the Bloch-McConnell equations. ${ }^{29}$ A detailed description is provided in the Supporting Information.

\section{ASSOCIATED CONTENT}

\section{sI Supporting Information}

The Supporting Information is available free of charge at https://pubs.acs.org/doi/10.1021/acs.jpclett.0c01322.

Samples of arginine and T4L99A sample preparations; a detailed list of experimental conditions for all NMR experiments, Table S1; detailed description of the NMR and computational methods; supporting tables with parameters derived and pulse sequences for the experiments performed;. supplementary Figures S1-S9 showing simulated MQ-CEST data; detailed NMR pulse sequence diagrams including for the ${ }^{1} \mathrm{H}$-detected sequence; a comparison of the sensitivity of ${ }^{13} \mathrm{C}$ and ${ }^{1} \mathrm{H}$ detected MQ-CEST spectra; all experimental data for T4L99A; and an error analysis of fitted parameters for T4L99A and analysis of the effects of relaxation rates on the results (PDF)

\section{AUTHOR INFORMATION}

\section{Corresponding Author}

D. Flemming Hansen - Institute of Structural and Molecular Biology, Division of Biosciences, University College London, London WC1E 6BT, United Kingdom; (1) orcid.org/00000003-0891-220X; Email: d.hansen@ucl.ac.uk

\section{Authors}

Gogulan Karunanithy - Institute of Structural and Molecular Biology, Division of Biosciences, University College London, London WC1E 6BT, United Kingdom; O orcid.org/00000002-8072-5911

Jochen Reinstein - Department of Biomolecular Mechanisms, Max Planck Institute for Medical Research, D-69120 Heidelberg, Germany

Complete contact information is available at:

https://pubs.acs.org/10.1021/acs.jpclett.0c01322

\section{Notes}

The authors declare no competing financial interest.

\section{ACKNOWLEDGMENTS}

Karola Gerecht is acknowledged for producing the sample of L99A T4L used in this study. The BBSRC (BB/R000255/1), the Wellcome Trust ( $\operatorname{ref} 101569 / \mathrm{z} / 13 / \mathrm{z}$ ), and the EPSRC are acknowledged for supporting the biomolecular NMR facility at University College London. Access to ultra-high-field NMR spectrometers was supported by the Francis Crick Institute through provision of access to the MRC Biomedical NMR Centre and by the University of Oxford Wellcome Institutional Strategic Support Fund, the John Fell Fund, as well as the Edward Penley Abraham Cephalosporin Fund, and the
Engineering and Physical Sciences Research Council (EP/ R029849/1). The Francis Crick Institute receives its core funding from Cancer Research UK (FC001029), the UK Medical Research Council (FC001029), and the Wellcome Trust (FC001029). This research is supported by the Leverhulme Trust (RPG-2016-268).

\section{REFERENCES}

(1) Karplus, M.; Kuriyan, J. Molecular Dynamics and Protein Function. Proc. Natl. Acad. Sci. U. S. A. 2005, 102, 6679-6685.

(2) Henzler-Wildman, K.; Kern, D. Dynamic Personalities of Proteins. Nature 2007, 450, 964-972.

(3) Alderson, T. R.; Kay, L. E. Unveiling Invisible Protein States with NMR Spectroscopy. Curr. Opin. Struct. Biol. 2020, 60, 39-49.

(4) Baldwin, A. J.; Kay, L. E. NMR Spectroscopy Brings Invisible Protein States into Focus. Nat. Chem. Biol. 2009, 5, 808-814.

(5) Vallurupalli, P.; Hansen, D. F.; Kay, L. E. Structures of Invisible, Excited Protein States by Relaxation Dispersion NMR Spectroscopy. Proc. Natl. Acad. Sci. U. S. A. 2008, 105, 11766-11771.

(6) Sauerwein, A. C.; Hansen, D. F. Relaxation Dispersion NMR Spectroscopy. In Protein NMR: Modern Techniques and Biomedical Applications; Springer: Boston, MA, 2015; pp 75-132.

(7) Jones, K. M.; Pollard, A. C.; Pagel, M. D. Clinical Applications of Chemical Exchange Saturation Transfer (CEST) MRI. J. Magn. Reson. Imaging 2018, 47, 11-27.

(8) Ling, W.; Eliav, U.; Navon, G.; Jerschow, A. Chemical Exchange Saturation Transfer by Intermolecular Double-Quantum Coherence. J. Magn. Reson. 2008, 194, 29-32.

(9) Yang, W.; Lee, J.; Leninger, M.; Windschuh, J.; Traaseth, N. J.; Jerschow, A. Magnetization Transfer in Liposome and Proteoliposome Samples That Mimic the Protein and Lipid Composition of Myelin. NMR Biomed. 2019, 32, No. e4097.

(10) Vallurupalli, P.; Bouvignies, G.; Kay, L. E. Studying "Invisible" Excited Protein States in Slow Exchange with a Major State Conformation. J. Am. Chem. Soc. 2012, 134, 8148-8161.

(11) Vallurupalli, P.; Sekhar, A.; Yuwen, T.; Kay, L. E. Probing Conformational Dynamics in Biomolecules via Chemical Exchange Saturation Transfer: A Primer. J. Biomol. NMR 2017, 67, 243-271.

(12) Forsén, S.; Hoffman, R. A. Study of Moderately Rapid Chemical Exchange Reactions by Means of Nuclear Magnetic Double Resonance. J. Chem. Phys. 1963, 39, 2892-2901.

(13) Borders, C. L.; Broadwater, J. A.; Bekeny, P. A.; Salmon, J. E.; Lee, A. S.; Eldridge, A. M.; Pett, V. B. A Structural Role for Arginine in Proteins: Multiple Hydrogen Bonds to Backbone Carbonyl Oxygens. Protein Sci. 1994, 3, 541-548.

(14) Pascal, S. M.; Yamazaki, T.; Singer, A. U.; Kay, L. E.; FormanKay, J. D. Structural and Dynamic Characterization of the Phosphotyrosine Binding Region of a Src Homology 2 DomainPhosphopeptide Complex by NMR Relaxation, Proton Exchange, and Chemical Shift Approaches. Biochemistry 1995, 34, 11353-11362.

(15) Friedt, J.; Leavens, F. M. V.; Mercier, E.; Wieden, H. J.; Kothe, U. An Arginine-Aspartate Network in the Active Site of Bacterial TruB Is Critical for Catalyzing Pseudouridine Formation. Nucleic Acids Res. 2014, 42, 3857-3870.

(16) Chong, P. A.; Vernon, R. M.; Forman-Kay, J. D. RGG/RG Motif Regions in RNA Binding and Phase Separation. J. Mol. Biol. 2018, 430, 4650-4665.

(17) Goldschen-Ohm, M. P.; Wagner, D. A.; Jones, M. V. Three Arginines in the GABAA Receptor Binding Pocket Have Distinct Roles in the Formation and Stability of Agonist- versus AntagonistBound Complexes. Mol. Pharmacol. 2011, 80, 647-656.

(18) Crowley, P. B.; Golovin, A. Cation- $\pi$ Interactions in ProteinProtein Interfaces. Proteins: Struct., Funct., Genet. 2005, 59, 231-239.

(19) Yoshimura, Y.; Oktaviani, N. A.; Yonezawa, K.; Kamikubo, H.; Mulder, F. A. A. Unambiguous Determination of Protein Arginine Ionization States in Solution by NMR Spectroscopy. Angew. Chem., Int. Ed. 2017, 56, 239-242. 
(20) Werbeck, N. D.; Kirkpatrick, J.; Hansen, D. F. Probing Arginine Side-Chains and Their Dynamics with Carbon-Detected NMR Spectroscopy: Application to the $42 \mathrm{KDa}$ Human Histone Deacetylase 8 at High PH. Angew. Chem., Int. Ed. 2013, 52, 31453147.

(21) Pritchard, R. B.; Hansen, D. F. Characterising Side Chains in Large Proteins by Protonless 13 C-Detected NMR Spectroscopy. Nat. Commun. 2019, 10, 1747 DOI: 10.1038/s41467-019-09743-4.

(22) Mackenzie, H. W.; Hansen, D. F. A 13C-Detected 15N Double-Quantum NMR Experiment to Probe Arginine Side-Chain Guanidinium 15N $\eta$ Chemical Shifts. J. Biomol. NMR 2017, 69, 123132.

(23) Gerecht, K.; Figueiredo, A. M.; Hansen, D. F. Determining Rotational Dynamics of the Guanidino Group of Arginine Side Chains in Proteins by Carbon-Detected NMR. Chem. Commun. 2017, 53, $10062-10065$.

(24) McCoy, M. A.; Mueller, L. Selective Shaped Pulse Decoupling in NMR: Homonuclear [13C]Carbonyl Decoupling. J. Am. Chem. Soc. 1992, 114, 2108-2112.

(25) Geen, H.; Freeman, R. Band-Selective Radiofrequency Pulses. J. Magn. Reson. 1991, 93, 93-141.

(26) McConnell, H. M. Reaction Rates by Nuclear Magnetic Resonance. J. Chem. Phys. 1958, 28, 430-431.

(27) Hansen, D. F.; Led, J. J. Implications of Using Approximate Bloch-McConnell Equations in NMR Analyses of Chemically Exchanging Systems: Application to the Electron Self-Exchange of Plastocyanin. J. Magn. Reson. 2003, 163, 215-227.

(28) Chakrabarti, K. S.; Ban, D.; Pratihar, S.; Reddy, J. G.; Becker, S.; Griesinger, C.; Lee, D. High-Power 1H Composite Pulse Decoupling Provides Artifact Free Exchange-Mediated Saturation Transfer (EST) Experiments. J. Magn. Reson. 2016, 269, 65-69.

(29) Vallurupalli, P.; Bouvignies, G.; Kay, L. E. A Computational Study of the Effects of $13 \mathrm{c}-13 \mathrm{c}$ Scalar Couplings on 13C CEST NMR Spectra: Towards Studies on a Uniformly 13C-Labeled Protein. ChemBioChem 2013, 14, 1709-1713.

(30) Farrow, N. A.; Zhang, O.; Forman-Kay, J. D.; Kay, L. E. A Heteronuclear Correlation Experiment for Simultaneous Determination of $15 \mathrm{~N}$ Longitudinal Decay and Chemical Exchange Rates of Systems in Slow Equilibrium. J. Biomol. NMR 1994, 4, 727-734.

(31) Liu, L.; Baase, W. A.; Matthews, B. W. Halogenated Benzenes Bound within a Non-Polar Cavity in T4 Lysozyme Provide Examples of I...S and I..Se Halogen-Bonding. J. Mol. Biol. 2009, 385, 595-605.

(32) Delaglio, F.; Grzesiek, S.; Vuister, G. W.; Zhu, G.; Pfeifer, J.; Bax, A. NMRPipe: A Multidimensional Spectral Processing System Based on UNIX Pipes. J. Biomol. NMR 1995, 6, 277-293.

(33) Lee, W.; Tonelli, M.; Markley, J. L. NMRFAM-SPARKY: Enhanced Software for Biomolecular NMR Spectroscopy. Bioinformatics 2015, 31, 1325-1327.

(34) Hansen, D. F.; Yang, D.; Feng, H.; Zhou, Z.; Wiesner, S.; Bai, Y.; Kay, L. E. An Exchange-Free Measure of $15 \mathrm{~N}$ Transverse Relaxation: An NMR Spectroscopy Application to the Study of a Folding Intermediate with Pervasive Chemical Exchange. J. Am. Chem. Soc. 2007, 129, 11468-11479. 